\title{
Establishment of sensitive and resistant variety of tomato on the basis of photosynthesis and antioxidative enzymes in the presence of cobalt applied as shotgun approach
}

\author{
Syed Aiman Hasan, Shamsul Hayat, Arif Shafi Wani, Aqil Ahmad \\ Plant Physiology Section, Department of Botany, Aligarh Muslim University, Aligarh 202 002, India \\ *Corresponding author: e-mail: shayat@lycos.com \\ Received: 13 April 2010; Accepted: 21 August 2011
}

\begin{abstract}
Cobalt (Co) affords both beneficial as well as toxic effects to plants. The present study was performed with an aim to find out the varietal differences among five tomato cultivars against the $\mathrm{Co}$ induced changes in growth, photosynthesis, nitrate reductase (E.C.1.6.6.1), carbonic anhydrase (E.C.4.2.1.1), antioxidative enzymes i.e. peroxidase (E.C.1.11.1.7), catalase (E.C.1.11.1.6), superoxide dismutase (E.C.1.15.1.1) and that of proline content. Seeds of tomato (varieties, K-25, NTS-9, NBR-Uday, Sarvodya, and Malti) were soaked in $0,100,200$ or $300 \mu \mathrm{M} \mathrm{CoCl}$ for $0,4,8,12 \mathrm{~h}$ (shotgun approach) and sampled at 30 days after sowing. All the varieties showed significantly different response to different treatment combinations. Despite substantial varietal difference, increased Co concentration caused concomitant decrease in growth, photosynthesis and the activity of nitrate reductase and carbonic anhydrase. However, the activity of antioxidant enzymes and that of proline content increased with the increased concentration of $\mathrm{Co}$ as well as duration of soaking in all the varieties. Out of the varieties, $\mathrm{K}-25$ possessed maximum antioxidative enzyme and proline content that represent its most resistant nature against the toxic effect of Co. The order of susceptibility/ sensitivity was K-25 > NTS-9 > NBR-Uday > Sarvodya > Malti.
\end{abstract}

Key words: antioxidative enzyme, carbonic anhydrase, cobalt, nitrate reductase, photosynthesis, tomato.

\section{INTRODUCTION}

Cobalt ( $\mathrm{C} 0$ ) is a natural earth element presented in trace amount in soil. Trace elements are necessary for normal metabolic functions in plants, but at higher concentration these metals are toxic and may severely interfere with physiological and biochemical functions (Jayakumar and Vijayarengan, 2006). Similarly, Co also affords both beneficial as well as harmful effects to plants. It has long been applied to plants to raise crops yield (Young, 1979). However, in excess concentration it caused a marked reduction in growth together with chlorosis and necrosis (Vanselow, 1966), inhibits photosynthesis (Van Asshe and Clijsters, 1990), seed germination and seedling growth (Dubey and Dwivedi, 1987). The activities of several enzymes are also disturbed by excessive amount of Co present within the plant (Shalygo et al., 1990) and thus finally reduced the quality of produce (Chatterjee et al., 2006). In general the average level of Co in the soil ranges 30-40 ppm (Kabata-Pendias and Pendias, 1991) and above that it generates toxicity. However, the 
concentration of $\mathrm{Co}$ in the soil is not the only factors that determine toxicity. Plant species vary in their sensitivity to $\mathrm{Co}$, soil type and soil chemistry greatly influence Co toxicity. One of the most important soil properties is soil acidity and the more acidic the soil, the greater the potential for Co toxicity, at any concentration. Keeping all these points in mind the present piece of work was designed, with an aim to find out the degree of tolerance among different varieties of tomato against differential concentration of $\mathrm{Co}_{0}$ applied as seed soaking i.e. shotgun approach (Hayat et al., 2010).

\section{MATERIAL AND METHODS}

Seeds of tomato (Lycopersicon esculentum) cv. K-25, NTS-9, NBR-Uday, Sarvodya, and Malti were purchased from National Seed Corporation Ltd., New Delhi, India. Healthy seeds were surface sterilized with $0.5 \%(\mathrm{v} / \mathrm{v})$ of sodium hypochlorite solution, followed by repeated washings with double distilled water and were soaked in $0,100,200$ or $300 \mu \mathrm{M}$ of Cobalt in the form of cobalt chloride for $0,4,8$ or $12 \mathrm{~h}$ (shotgun approach). These treated seeds were sown in sand, moistened with deionized water in plastic pots of 6 inch diameter. These pots were kept in a plant growth chamber illuminated by incandescent light, with day/night temperature of $25 \pm 2^{\circ} \mathrm{C}$ on $14 / 10 \mathrm{~h}$ photoperiod. The humidity was maintained at $65 \pm 5 \%$. The plants after their germination in dark for 5 days were supplied with $50 \mathrm{ml}$ of nutrient solution. The composition of nutrient solution was $0.5 \mu \mathrm{M} \mathrm{KNO}, 0.5 \mu \mathrm{M}$ Ca $\left(\mathrm{NO}_{3}\right)_{2}, 0.5 \mu \mathrm{M} \mathrm{MgSO}_{4}$, $2.5 \mu \mathrm{M} \mathrm{KH}_{2} \mathrm{PO}_{4}, 2 \mu \mathrm{M} \mathrm{NH}{ }_{4} \mathrm{Cl}, 100 \mu \mathrm{M}$ Fe-K-EDTA, $30 \mu \mathrm{M}$

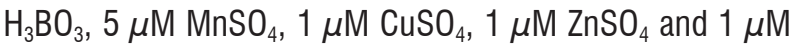
$\left(\mathrm{NH}_{4}\right)_{6} \mathrm{Mo}_{7} \mathrm{O}_{24}$ per liter. The $\mathrm{pH}$ of the nutrient solution was adjusted to 5.0 with $\mathrm{HCl}$. The design of the experiment was complete randomized and the position of pots, in the growth chamber was changed daily. The plants were sampled at 30 days after sowing (DAS) to make the various observations. The plants were uprooted and washed under running tap water. The shoot was separated from roots and was weighed for their fresh mass. These were subsequently transferred to an oven run at $80{ }^{\circ} \mathrm{C}$ and left there for 48 hours, and weighed again to obtain their dry mass. Leaf area was determined by gravimetric method where the leaf area of randomly selected leaves from each treatment, was determined by tracing its outline on the graph sheet.

The chlorophyll content in the leaves was measured with the help of a Minolta chlorophyll meter (SPAD-502, Konica Minolta Sensing Inc. Japan). Leaf water potential, was measured in fresh, detached leaves of the sample plants by using PSYPRO, water potential system (WESCOR, Inc. Longman, USA).The rate of photosynthesis and internal $\mathrm{CO}_{2}$ concentration were measured by using a LI-6400 portable photosynthetic system (LI-COR, Lincoln, NE, USA). The measurements were made in the uppermost fully expanded leaves, between 1100 and 1300 hours.

The activity of NR was determined in fresh leaf samples by the procedure explained by Jaworski (1971). The fresh leaf samples were cut into small pieces and transferred to plastic vials, containing phosphate buffer ( $\mathrm{pH} 7.5)$ followed by the addition of potassium nitrate and isopropanol solutions. The reaction mixture was incubated at $30^{\circ} \mathrm{C}$ for $2 \mathrm{~h}$ followed by addition of sulfanilamide and $\mathrm{N}-1$ - naphthylethylenediamine dihydrochloride. The absorbance of the colour was read at $540 \mathrm{~nm}$ and was compared with that of the calibration curve.

The activity of CA was determined following the procedure described by Dwivedi and Randhawa (1974). The leaf samples were cut into small pieces and suspended in cystein hydrochloride solution. The samples were incubated at $4{ }^{\circ} \mathrm{C}$ for $20 \mathrm{~min}$. The pieces were blotted and transferred to the test tubes, containing phosphate buffer (pH 6.8) followed by the addition of alkaline bicarbonate solution and bromothymol blue indicator. The test tube was incubated at $5^{\circ} \mathrm{C}$ for $20 \mathrm{~min}$. The reaction mixture was titrated against $0.01 \mathrm{~mol} / \mathrm{L} \mathrm{HCl}$, after addition of $0.2 \mathrm{~mL}$ of methyl red indicator.

For the estimation of antioxidative enzymes, the leaf tissue $(0.5 \mathrm{~g})$ was homogenized in $50 \mathrm{mmol} / \mathrm{L}$ phosphate buffer (pH 7.0) containing $1 \%(\mathrm{w} / \mathrm{v})$ soluble polyvinylpyrrolidone. The homogenate was centrifuged at $15000 \mathrm{~g}$ for $10 \mathrm{~min}$ at $4^{\circ} \mathrm{C}$ and the supernatant was used as a source of the enzymes catalase (CAT), peroxidase (POX) and superoxide dismutase (SOD). CAT and POX were assayed following the procedure described by Chance and Maehly (1955). CAT was estimated by titrating the reaction mixture, consisting of phosphate buffer ( $\mathrm{pH} 6.8$ ), $0.1 \mathrm{~mol} / \mathrm{L} \mathrm{H}_{2} \mathrm{O}_{2}$, enzyme extract and $2 \% \mathrm{H}_{2} \mathrm{SO}_{4}$, against $0.1 \mathrm{~mol} / \mathrm{L}$ potassium permanganate 
solution. The reaction mixture for peroxidase consisted of pyrogallol, phosphate buffer ( $\mathrm{pH} 6.8$ ), $1 \% \mathrm{H}_{2} \mathrm{O}_{2}$ and enzyme extract. Change in absorbance due to catalytic conversion to pyrogallol to perpurogalline was noted at an interval of $20 \mathrm{~s}$ for 2 min at $420 \mathrm{~nm}$ on a spectrophotometer. A control set was prepared by using DDW instead of enzyme extract. The activity of superoxide dismutase was assayed by measuring its ability to inhibit the photochemical reduction of nitroblue tetrazolium following the method of Beauchamp and Fridovich (1971). The reaction mixture contained $50 \mathrm{mmol} / \mathrm{L}$ phosphate buffer (pH 7.8), $13 \mathrm{mmol} / \mathrm{L}$ ethylenediaminetetraacetic acid and 0-50 $\mathrm{mL}$ enzyme extract and was placed under a $15 \mathrm{~W}$ fluorescent lamp. The reaction was started by switching on the light and was allowed to run for $10 \mathrm{~min}$. The reaction was stopped by switching off the light. Fifty per cent inhibition by light was considered as 1 enzyme unit.

The proline content in fresh leaf and root samples was determined by adopting the method of Bates et al. (1973). Samples were extracted with sulfosalicylic acid and an equal volume of glacial acetic acid and ninhydrine solutions were added. The samples were heated at $100{ }^{\circ} \mathrm{C}$, to which $5 \mathrm{~mL}$ of toluene was added. The absorbance of the toluene layer was read at $528 \mathrm{~nm}$ on a spectrophotometer.

Data were statistically analyzed using analysis of variance (Anova) by SPSS (ver. 10; SPSS Inc., Chicago, IL, USA). The least significant difference was calculated for the significant data at $P<0.05$.

\section{RESULTS}

Growth parameters: All the varieties exhibited significantly different response to different concentration of metal as well as duration of soaking. Pre sowing seed soaking treatment caused significant decrease in the fresh and dry mass of shoot and leaf area. The decrease was proportionate to the concentration of the metal $(100,200$ or $300 \mu \mathrm{M})$ as well as duration of soaking (4, 8 or $12 \mathrm{~h})$. The highest concentration of metal $(300 \mu \mathrm{M})$ and longest duration of soaking i.e. $12 \mathrm{~h}$ caused maximum damage. The variety K-25 was found to be most resistant (Tables 1-3). The order of susceptibility/sensitivity was K-25 > NTS- $9>$ NBR-Uday $>$ Sarvodya $>$ Malti.

Table 1. Effect of pre sowing seed soaking treatment of cobalt (100, 200 or $300 \mu \mathrm{M})$ for 4,8 or $12 \mathrm{~h}$ on the shoot fresh mass (g) in five varieties of tomato $(L$. esculentum Mill.) at 30 days AS.

\begin{tabular}{|c|c|c|c|c|c|c|c|c|}
\hline \multirow{2}{*}{$\begin{array}{l}\text { Duration of } \\
\text { Seed soaking } \\
\text { in } C_{0}(\mu \mathrm{M})\end{array}$} & \multirow{2}{*}{ Treatments } & \multicolumn{6}{|c|}{ Varieties } & \multirow{2}{*}{ LSD at $5 \%$} \\
\hline & & $\mathrm{K}-25$ & NTS-9 & NBR-Uday & Sarvodya & Malti & Mean & \\
\hline \multicolumn{2}{|c|}{ Unsoaked control } & 3.10 & 2.52 & 2.21 & 1.89 & 1.80 & 2.30 & \multirow{6}{*}{$\begin{array}{r}V=0.14 \\
C=0.20 \\
V x C=0.39\end{array}$} \\
\hline \multirow{5}{*}{$4 \mathrm{~h}$} & 0 & 3.19 & 2.55 & 2.24 & 2.00 & 1.87 & 2.37 & \\
\hline & 100 & 2.94 & 2.32 & 2.02 & 1.79 & 1.50 & 2.11 & \\
\hline & 200 & 2.25 & 2.11 & 1.49 & 1.40 & 1.31 & 1.71 & \\
\hline & 300 & 2.00 & 1.70 & 1.26 & 1.19 & 1.09 & \multirow[t]{2}{*}{1.44} & \\
\hline & Mean & 2.59 & 2.17 & 1.75 & 1.59 & 1.44 & & \\
\hline \multirow{5}{*}{$8 \mathrm{~h}$} & 0 & 3.22 & 2.59 & 2.28 & 2.06 & 1.89 & 2.40 & \multirow{5}{*}{$\begin{array}{r}V=0.25 \\
C=0.10 \\
V x C=0.39\end{array}$} \\
\hline & 100 & 2.80 & 2.00 & 1.86 & 1.50 & 1.39 & 1.91 & \\
\hline & 200 & 1.84 & 1.70 & 1.39 & 1.11 & 1.00 & 1.40 & \\
\hline & 300 & 1.77 & 1.64 & 1.23 & 1.00 & 0.90 & 1.30 & \\
\hline & Mean & 2.40 & 1.98 & 1.69 & 1.41 & 1.29 & & \\
\hline \multirow{5}{*}{$12 \mathrm{~h}$} & 0 & 3.29 & 2.58 & 2.30 & 2.08 & 1.70 & 2.39 & \multirow{5}{*}{$\begin{array}{c}V=0.18 \\
C=0.20 \\
V x C=0.42\end{array}$} \\
\hline & 100 & 2.46 & 1.83 & 1.60 & 1.30 & 1.20 & 1.67 & \\
\hline & 200 & 1.66 & 1.50 & 1.16 & 1.00 & 0.89 & 1.24 & \\
\hline & 300 & 1.40 & 1.23 & 1.04 & 0.80 & 0.64 & 1.02 & \\
\hline & Mean & 2.20 & 1.78 & 1.52 & 1.29 & 1.10 & & \\
\hline
\end{tabular}

$A S$, after sowing; $V$, varieties; $C$, cobalt concentration. 
Table 2. Effect of pre sowing seed soaking treatment of cobalt $(100,200$ or $300 \mu \mathrm{M})$ for 4,8 or $12 \mathrm{~h}$ on the shoot dry mass (g) in five varieties of tomato ( $\mathrm{L}$. esculentum Mill.) at 30 days AS.

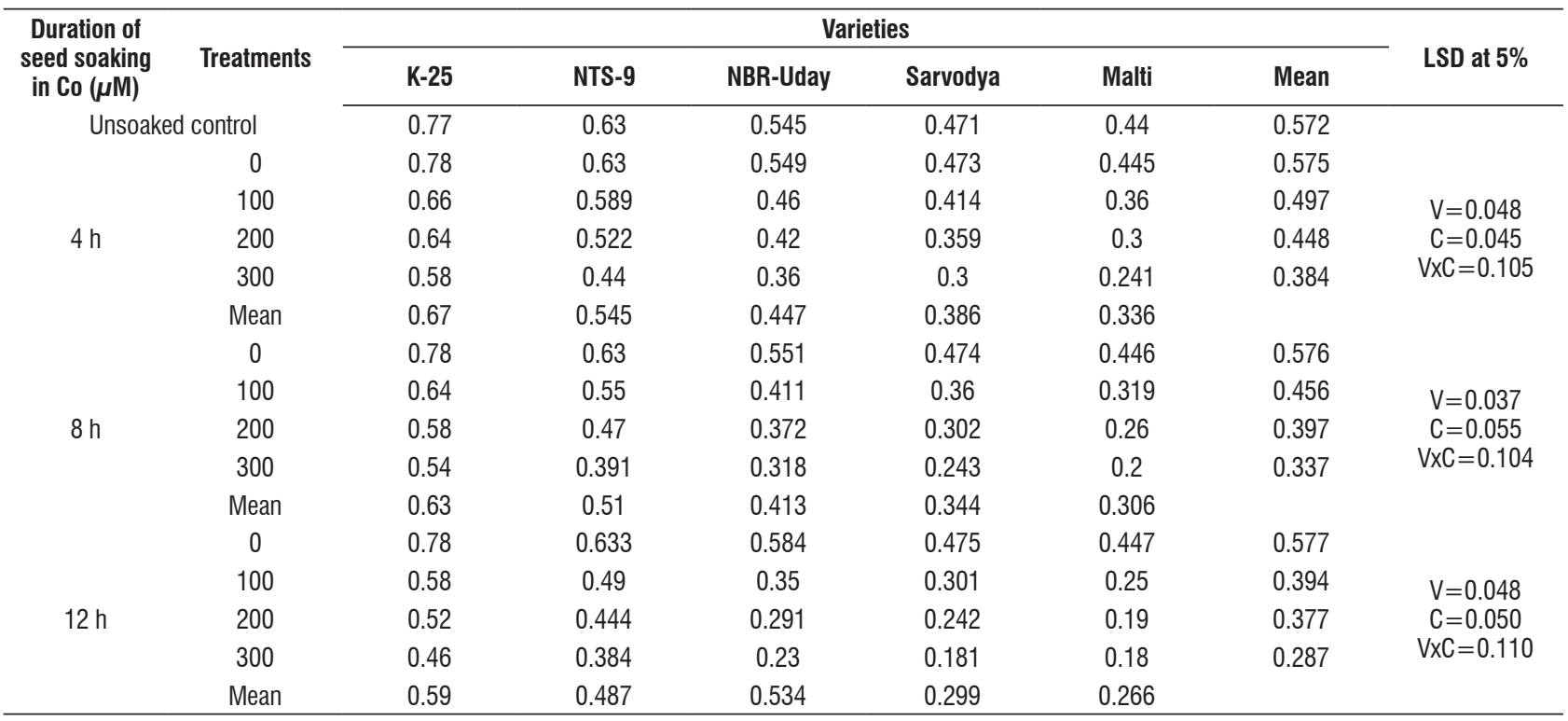

$A S$, after sowing; $V$, varieties; $C$, cobalt concentration.

Table 3. Effect of pre sowing seed soaking treatment of cobalt $(100,200$ or $300 \mu \mathrm{M})$ for 4,8 or $12 \mathrm{~h}$ on the leaf area ( $\left.\mathrm{cm}^{2}\right)$ in five varieties of tomato (L. esculentum Mill.) at 30 days AS.

\begin{tabular}{|c|c|c|c|c|c|c|c|c|}
\hline \multirow{2}{*}{$\begin{array}{l}\text { Duration of } \\
\text { seed soaking } \\
\text { in Co }(\mu \mathrm{M})\end{array}$} & \multirow{2}{*}{ Treatments } & \multicolumn{6}{|c|}{ Varieties } & \multirow{2}{*}{ LSD at $5 \%$} \\
\hline & & K-25 & NTS-9 & NBR-Uday & Sarvodya & Malti & Mean & \\
\hline \multicolumn{2}{|c|}{ Unsoaked control } & 5.79 & 5.68 & 5.4 & 5.28 & 4.81 & 5.39 & \multirow{6}{*}{$\begin{array}{c}V=0.17 \\
C=0.55 \\
V x C=0.81\end{array}$} \\
\hline \multirow{5}{*}{$4 \mathrm{~h}$} & 0 & 5.82 & 5.71 & 5.43 & 5.3 & 4.85 & 5.42 & \\
\hline & 100 & 5.64 & 5.59 & 5.17 & 5 & 4.7 & 5.22 & \\
\hline & 200 & 5 & 4.8 & 4.6 & 3.97 & 3.4 & 4.35 & \\
\hline & 300 & 4.16 & 3.8 & 3.44 & 2.82 & 2.36 & \multirow[t]{2}{*}{3.31} & \\
\hline & Mean & 5.15 & 4.97 & 4.66 & 4.27 & 3.82 & & \\
\hline \multirow{5}{*}{$8 \mathrm{~h}$} & 0 & 5.84 & 5.74 & 5.46 & 5.32 & 4.86 & 5.44 & \multirow{5}{*}{$\begin{array}{c}V=0.14 \\
C=0.20 \\
V x C=0.14\end{array}$} \\
\hline & 100 & 5.58 & 5.51 & 5.1 & 4.96 & 4.66 & 5.16 & \\
\hline & 200 & 4.32 & 4.11 & 3.92 & 3.6 & 3.17 & 3.82 & \\
\hline & 300 & 3.71 & 3.5 & 3.3 & 3 & 2.56 & 3.21 & \\
\hline & Mean & 4.86 & 4.71 & 4.77 & 4.22 & 3.79 & & \\
\hline \multirow{5}{*}{$12 \mathrm{~h}$} & 0 & 5.87 & 5.76 & 5.47 & 5.34 & 4.87 & 5.46 & \multirow{5}{*}{$\begin{array}{c}V=0.14 \\
C=0.30 \\
V x C=0.50\end{array}$} \\
\hline & 100 & 5.55 & 5.47 & 5.06 & 4.91 & 4.6 & 5.11 & \\
\hline & 200 & 4.06 & 3.86 & 3.61 & 3.42 & 2.93 & 3.97 & \\
\hline & 300 & 3.5 & 3.28 & 2.97 & 2.69 & 2.4 & 2.96 & \\
\hline & Mean & 4.74 & 4.59 & 4.27 & 4.09 & 3.7 & & \\
\hline
\end{tabular}

$A S$, after sowing; $V$, varieties; $C$, cobalt concentration.

Leaf water potential $(\psi)$ and SPAD chlorophyll value: It is evident from table 4 and 5 that the pre sowing seed soaking treatment caused a significant decrease in leaf water potential and SPAD chlorophyll values of the resulting plants. The highest concentration of metal $(300 \mu \mathrm{M})$ generate maximum inhibition at all the soaking duration (4, 8 or $12 \mathrm{~h}$ ) irrespective 
of varietal difference and decreased the values of $\psi$ by $75.0 \%$, $93.3 \%$ and $109.8 \% ; 67.1 \%, 88.4 \%$ and $100 \% ; 50.6 \%, 85.0 \%$ and $105.0 \% ; 64.2 \%, 96.4 \%$ and $111.7 \% ; 64.7 \%, 98.8 \%$ and $114.7 \%$ and that of SPAD chlorophyll by $25.3 \%, 31.3 \%$ and
47.1\%; $30.1 \%, 37.6 \%$ and $50.4 \% ; 36.8 \%, 42.9 \%$ and $57.9 \%$ $38.8 \%, 44.8 \%$ and $52.5 \% ; 40.1 \%, 47.4 \%$ and $59.8 \%$ in $\mathrm{K}-25$, NTS-9, NBR-Uday, Sarvodya and Malti where seeds were pretreated with 4,8 or $12 \mathrm{~h}$ respectively.

Table 4. Effect of pre sowing seed soaking treatment of cobalt $(100,200$ or $300 \mu \mathrm{M})$ for 4,8 or $12 \mathrm{~h}$ on the leaf water potential (Mpa) in five varieties of tomato (L. esculentum Mill.) at 30 days AS.

\begin{tabular}{|c|c|c|c|c|c|c|c|c|}
\hline \multirow{2}{*}{$\begin{array}{l}\text { Duration of } \\
\text { seed soaking } \\
\text { in Co }(\mu \mathrm{M})\end{array}$} & \multirow{2}{*}{ Treatments } & \multicolumn{6}{|c|}{ Varieties } & \multirow{2}{*}{ LSD at $5 \%$} \\
\hline & & $\mathrm{K}-25$ & NTS-9 & NBR-Uday & Sarvodya & Malti & Mean & \\
\hline \multicolumn{2}{|c|}{ Unsoaked control } & -0.6 & -0.69 & -0.78 & -0.8 & -0.82 & -0.73 & \multirow{6}{*}{$\begin{array}{c}V=0.06 \\
C=0.12 \\
V x C=0.20\end{array}$} \\
\hline \multirow{5}{*}{$4 \mathrm{~h}$} & 0 & -0.6 & -0.7 & -0.81 & -0.84 & -0.88 & -0.76 & \\
\hline & 100 & -0.7 & -0.85 & -0.97 & -1.08 & -1.12 & -0.95 & \\
\hline & 200 & -0.9 & -1.04 & -1.13 & -1.21 & -1.32 & -1.11 & \\
\hline & 300 & -1.1 & -1.17 & -1.22 & -1.38 & -1.45 & \multirow[t]{2}{*}{-1.25} & \\
\hline & Mean & -0.8 & -0.94 & -1.03 & -1.12 & -1.19 & & \\
\hline \multirow{5}{*}{$8 \mathrm{~h}$} & 0 & -0.6 & -0.69 & -0.8 & -0.84 & -0.89 & -0.76 & \multirow{5}{*}{$\begin{array}{c}V=0.08 \\
C=0.18 \\
V x C=0.29\end{array}$} \\
\hline & 100 & -0.8 & -0.91 & -1.04 & -1.21 & -1.33 & -1.05 & \\
\hline & 200 & -1 & -1.22 & -1.23 & -1.43 & -1.5 & -1.25 & \\
\hline & 300 & -1.2 & -1.3 & -1.48 & -1.65 & -1.77 & -1.47 & \\
\hline & Mean & -0.9 & -1.03 & -1.13 & -1.28 & -1.34 & & \\
\hline \multirow{5}{*}{$12 \mathrm{~h}$} & 0 & -0.6 & -0.7 & -0.79 & -0.85 & -0.88 & -0.76 & \multirow{5}{*}{$\begin{array}{c}V=0.06 \\
C=0.17 \\
V x C=0.26\end{array}$} \\
\hline & 100 & -0.8 & -0.96 & -1.19 & -1.4 & -1.51 & -1.18 & \\
\hline & 200 & -1.1 & -1.17 & -1.41 & -1.63 & -1.7 & -1.39 & \\
\hline & 300 & -1.3 & -1.4 & -1.62 & -1.8 & -1.89 & -1.59 & \\
\hline & Mean & -0.9 & -1.05 & -1.25 & -1.42 & -1.49 & & \\
\hline
\end{tabular}

$A S$, after sowing; $V$, varieties; $C$, cobalt concentration.

Table 5. Effect of3 pre sowing seed soaking treatment of cobalt $(100,200$ or $300 \mu \mathrm{M})$ for 4,8 or $12 \mathrm{~h}$ on the SPAD chlorophyll in five varieties of tomato $(L$. esculentum Mill.) at 30 days AS.

\begin{tabular}{|c|c|c|c|c|c|c|c|c|}
\hline \multirow{2}{*}{$\begin{array}{l}\text { Duration of } \\
\text { seed soaking } \\
\text { in Co }(\mu \mathrm{M})\end{array}$} & \multirow{2}{*}{ Treatments } & \multicolumn{6}{|c|}{ Varieties } & \multirow{2}{*}{ LSD at $5 \%$} \\
\hline & & K-25 & NTS-9 & NBR-Uday & Sarvodya & Malti & Mean & \\
\hline \multicolumn{2}{|c|}{ Unsoaked control } & 42.1 & 34.2 & 28.6 & 26 & 24.7 & 31.12 & \multirow{6}{*}{$\begin{array}{r}V=1.50 \\
C=3.00 \\
V x C=5.15\end{array}$} \\
\hline \multirow{5}{*}{$4 \mathrm{~h}$} & 0 & 43.1 & 35 & 29 & 27 & 25.06 & 31.83 & \\
\hline & 100 & 40 & 31.91 & 26.4 & 23.6 & 22 & 28.78 & \\
\hline & 200 & 36.8 & 27.9 & 22 & 20 & 18.41 & 25.02 & \\
\hline & 300 & 32.2 & 24.44 & 18.32 & 16.52 & 15 & \multirow[t]{2}{*}{21.29} & \\
\hline & Mean & 38 & 29.81 & 23.93 & 21.78 & 20.11 & & \\
\hline \multirow{5}{*}{$8 \mathrm{~h}$} & 0 & 43.7 & 35.6 & 29.8 & 27.2 & 25.09 & 32.27 & \multirow{5}{*}{$\begin{array}{r}V=1.88 \\
C=3.19 \\
V x C=5.68\end{array}$} \\
\hline & 100 & 37.5 & 30 & 24.8 & 22.17 & 22.11 & 26.91 & \\
\hline & 200 & 35 & 25 & 19.97 & 18.25 & 16.6 & 22.96 & \\
\hline & 300 & 30 & 22.2 & 17 & 15 & 13.19 & 19.47 & \\
\hline & Mean & 36.6 & 28.2 & 22.89 & 20.56 & 18.74 & & \\
\hline \multirow{5}{*}{$12 \mathrm{~h}$} & 0 & 44.1 & 36 & 30 & 37.8 & 25.16 & 32.61 & \multirow{5}{*}{$\begin{array}{c}V=1.45 \\
C=3.25 \\
V x C=5.37\end{array}$} \\
\hline & 100 & 32.7 & 28.4 & 22.5 & 20 & 16.6 & 23.92 & \\
\hline & 200 & 27 & 23.11 & 17 & 15.19 & 11 & 18.66 & \\
\hline & 300 & 23.3 & 17.84 & 12.62 & 13.18 & 10.11 & 15.4 & \\
\hline & Mean & 31.8 & 26.33 & 20.53 & 19.04 & 15.56 & & \\
\hline
\end{tabular}

$A S$, after sowing; $V$, varieties; $C$, cobalt concentration. 
Net Photosynthetic rate $\left(\mathrm{P}_{\mathrm{N}}\right)$ and internal $\mathrm{CO}_{2}$ concentration (Ci): The pre sowing seed soaking in different concentration of $\mathrm{Co}$ significantly reduced the $\mathrm{P}_{\mathrm{N}}$ and $\mathrm{Ci}$ (Tables 6-7) in all the varieties. However, the varieties showed significantly different response to different concentration of metal as well as duration of soaking. Out of the three $\mathrm{Co}_{0}$ concentrations, the $100 \mu \mathrm{M}$ was found to be least toxic, irrespective of duration of soaking and did not generate any significant response for $\mathrm{Ci}$ in all the varieties. Among the varieties, $\mathrm{K}-25$ showed maximum resistance against all the Co concentrations as well as duration of soaking. The highest concentration of metal $300 \mu \mathrm{M}$ and longest duration of soaking (12 h) was most detrimental for most of the varieties for $\mathrm{P}_{\mathrm{N}}$ and $\mathrm{Ci}$.

Table 6. Effect of pre sowing seed soaking treatment of cobalt $(100,200$ or $300 \mu \mathrm{M})$ for 4,8 or $12 \mathrm{~h}$ on the $\mathrm{P}_{\mathrm{N}}\left(\mu \mathrm{MCO}_{2} \mathrm{~m}^{-2} \mathrm{~S}^{-1}\right)$ in five varieties of tomato $(L$. esculentum Mill.) at 30 days AS.

\begin{tabular}{|c|c|c|c|c|c|c|c|c|}
\hline \multirow{2}{*}{$\begin{array}{l}\text { Duration of } \\
\text { seed soaking } \\
\text { in } C_{0}(\mu \mathrm{M})\end{array}$} & \multirow{2}{*}{ Treatments } & \multicolumn{6}{|c|}{ Varieties } & \multirow{2}{*}{ LSD at $5 \%$} \\
\hline & & K-25 & NTS-9 & NBR-Uday & Sarvodya & Malti & Mean & \\
\hline \multicolumn{2}{|c|}{ Unsoaked control } & 9.1 & 7.48 & 6.81 & 5.52 & 4.73 & 6.72 & \multirow{6}{*}{$\begin{array}{c}V=0.75 \\
C=0.48 \\
V x C=1.48\end{array}$} \\
\hline \multirow{5}{*}{$4 \mathrm{~h}$} & 0 & 9 & 7.59 & 6.88 & 5.57 & 4.77 & 6.76 & \\
\hline & 100 & 8.59 & 7.14 & 6.12 & 5 & 4.12 & 6.19 & \\
\hline & 200 & 8.51 & 6.42 & 5.69 & 4.29 & 3.6 & 5.7 & \\
\hline & 300 & 7.92 & 5.96 & 5.2 & 3.86 & 3.1 & 5.2 & \\
\hline & Mean & 8.5 & 6.77 & 5.97 & 4.68 & 3.89 & & \\
\hline \multirow{5}{*}{$8 \mathrm{~h}$} & 0 & 9.04 & 8.02 & 6.9 & 5.6 & 4.79 & 6.87 & \multirow{5}{*}{$\begin{array}{c}V=0.73 \\
C=0.58 \\
V x C=1.41\end{array}$} \\
\hline & 100 & 8 & 6.66 & 5.9 & 4.88 & 4 & 5.88 & \\
\hline & 200 & 7.5 & 6.14 & 5.4 & 4 & 3.35 & 5.27 & \\
\hline & 300 & 6.93 & 5.61 & 4.85 & 3.6 & 2.8 & 4.75 & \\
\hline & Mean & 7.86 & 6.6 & 5.76 & 4.52 & 3.73 & & \\
\hline \multirow{5}{*}{$12 \mathrm{~h}$} & 0 & 9.1 & 8.06 & 6.94 & 5.66 & 4.82 & 6.91 & \multirow{5}{*}{$\begin{aligned} V & =0.36 \\
C & =0.58 \\
V x C & =1.04\end{aligned}$} \\
\hline & 100 & 7.6 & 6.19 & 5.7 & 4.17 & 3.8 & 5.49 & \\
\hline & 200 & 7 & 5.42 & 5 & 3.7 & 3.1 & 4.82 & \\
\hline & 300 & 6.55 & 4.94 & 4.56 & 3.11 & 2.49 & 4.33 & \\
\hline & Mean & 7.56 & 6.15 & 5.55 & 4.16 & 3.55 & & \\
\hline
\end{tabular}

$A S$, after sowing; $V$, varieties; $C$, cobalt concentration.

Table 7. Effect of pre sowing seed soaking treatment of cobalt $(100,200$ or $300 \mu \mathrm{M})$ for 4,8 or $12 \mathrm{~h}$ on the leaf Ci (ppm) in five varieties of tomato (L. esculentum Mill.) at 30 days AS.

\begin{tabular}{|c|c|c|c|c|c|c|c|c|}
\hline \multirow{2}{*}{$\begin{array}{l}\text { Duration of } \\
\text { seed soaking } \\
\text { in Co }(\mu \mathrm{M})\end{array}$} & \multirow{2}{*}{ Treatments } & \multicolumn{6}{|c|}{ Varieties } & \multirow{2}{*}{ LSD at $\mathbf{5} \%$} \\
\hline & & K-25 & NTS-9 & NBR-Uday & Sarvodya & Malti & Mean & \\
\hline \multicolumn{2}{|c|}{ Unsoaked control } & 310 & 284 & 280 & 273 & 251 & 279 & \\
\hline \multirow{5}{*}{$4 \mathrm{~h}$} & 0 & 312 & 286 & 280 & 277 & 254 & 281 & \\
\hline & 100 & 310 & 282 & 280 & 274 & 251 & 279 & $V=6.00$ \\
\hline & 200 & 290 & 261 & 256 & 240 & 229 & 255 & $C=20.00$ \\
\hline & 300 & 270 & 239 & 222 & 218 & 207 & 231 & $V x C=30.00$ \\
\hline & Mean & 295 & 267 & 259 & 252 & 235 & & \\
\hline \multirow{5}{*}{$8 \mathrm{~h}$} & 0 & 316 & 289 & 281 & 279 & 256 & 284 & \\
\hline & 100 & 313 & 286 & 280 & 277 & 253 & 281 & $V=7.00$ \\
\hline & 200 & 280 & 247 & 230 & 221 & 212 & 238 & $C=25.00$ \\
\hline & 300 & 242 & 201 & 200 & 208 & 200 & 210 & $V x C=36.00$ \\
\hline & Mean & 288 & 255 & 245 & 246 & 230 & & \\
\hline \multirow{5}{*}{$12 \mathrm{~h}$} & 0 & 318 & 291 & 283 & 280 & 258 & 286 & \\
\hline & 100 & 312 & 289 & 277 & 275 & 251 & 280 & $V=6.00$ \\
\hline & 200 & 255 & 224 & 221 & 200 & 189 & 217 & $C=21.00$ \\
\hline & 300 & 220 & 201 & 190 & 186 & 180 & 195 & $V x C=30.00$ \\
\hline & Mean & 276 & 251 & 242 & 235 & 219 & & \\
\hline
\end{tabular}

$A S$, after sowing; $V$, varieties; $C$, cobalt concentration. 
Nitrate reductase (NR) and carbonic anhydrase (CA) activities: The leaves of the plants raised from the seeds given pre sowing seed soaking treatment in Co possessed significantly lower NR and CA activities (Tables 8-9). Degree of damage caused by the metal was directly proportional to the concentration of metal as well as soaking duration. The lowest duration of soaking i.e. $4 \mathrm{~h}$ in 100,200 or $300 \mu \mathrm{M}$ of Co decreased the activity of NR by $5.6 \%, 11.9 \%$ and $19.4 \%$;
$8.4 \%, 15.2 \%$ and $21.6 \% ; 8.6 \%, 17.9 \%$ and $22.5 \% ; 10.4 \%$, $19.2 \%$ and $24.0 \% ; 10.5 \%, 22.7 \%$ and $30.4 \%$ and that of $\mathrm{CA}$ by $6.5 \%, 17.7 \%$ and $22.1 \% ; 7.6 \%, 20.4 \%$ and $34.1 \% ; 17.5 \%$, $26.7 \%$ and $38.0 \% ; 14.4 \%, 18.0 \%$ and $40.0 \% ; 19.2 \%, 40.8 \%$ and $46.5 \%$ in K-25, NTS-9, NBR-Uday, Sarvodya and Malti respectively over their respective controls. The activity of both enzymes decreased further as the duration of soaking was extended.

Table 8. Effect of pre sowing seed soaking treatment of cobalt $(100,200$ or $300 \mu \mathrm{M})$ for 4,8 or $12 \mathrm{~h}$ on the leaf NR activity $\left(\mathrm{n} \mathrm{MNO}_{2} \mathrm{~g}^{-1} \mathrm{~h}^{-1} \mathrm{~F} . \mathrm{M}\right.$.) in five varieties of tomato (L. esculentum Mill.) at 30 days AS.

\begin{tabular}{|c|c|c|c|c|c|c|c|c|}
\hline \multirow{2}{*}{$\begin{array}{l}\text { Duration of } \\
\text { seed soaking } \\
\text { in Co }(\mu \mathrm{M})\end{array}$} & \multirow{2}{*}{ Treatments } & \multicolumn{6}{|c|}{ Varieties } & \multirow{2}{*}{ LSD at $5 \%$} \\
\hline & & K-25 & NTS-9 & NBR-Uday & Sarvodya & Malti & Mean & \\
\hline \multicolumn{2}{|c|}{ Unsoaked control } & 317 & 291 & 273 & 250 & 242 & 274 & \\
\hline & 0 & 318 & 295 & 279 & 254 & 246 & 278 & \\
\hline & 100 & 300 & 270 & 255 & 230 & 200 & 251 & $V=16.00$ \\
\hline \multirow{5}{*}{$4 \mathrm{~h}$} & 200 & 280 & 250 & 229 & 205 & 190 & 230 & $C=15.00$ \\
\hline & 300 & 256 & 231 & 216 & 193 & 171 & 213 & $V x C=35.80$ \\
\hline & Mean & 288 & 261 & 244 & 220 & 201 & & \\
\hline & 0 & 320 & 297 & 281 & 256 & 249 & 280 & \\
\hline & 100 & 294 & 255 & 231 & 208 & 190 & 235 & $V=15.00$ \\
\hline \multirow[t]{5}{*}{$8 \mathrm{~h}$} & 200 & 269 & 234 & 209 & 180 & 160 & 210 & $C=11.00$ \\
\hline & 300 & 237 & 229 & 200 & 170 & 149 & 197 & $V x C=30.00$ \\
\hline & Mean & 280 & 253 & 230 & 203 & 187 & & \\
\hline & 0 & 321 & 299 & 281 & 257 & 250 & 281 & \\
\hline & 100 & 279 & 240 & 228 & 185 & 177 & 221 & $V=20.00$ \\
\hline \multirow[t]{3}{*}{$12 \mathrm{~h}$} & 200 & 253 & 220 & 195 & 162 & 146 & 195 & $\mathrm{C}=15.00$ \\
\hline & 300 & 225 & 211 & 180 & 150 & 132 & 179 & $V x C=40.00$ \\
\hline & Mean & 269 & 242 & 221 & 187 & 176 & & \\
\hline
\end{tabular}

$A S$, after sowing; $V$, varieties; $C$, cobalt concentration.

Table 9. Effect of pre sowing seed soaking treatment of cobalt $(100,200$ or $300 \mu \mathrm{M})$ for 4,8 or $12 \mathrm{~h}$ on the leaf CA activity $\left(\mathrm{mol} \mathrm{CO}_{2} \mathrm{~kg}^{-1}\left(\mathrm{~F}_{\mathrm{M}} \mathrm{M}\right.\right.$.) s $\left.\mathrm{s}^{-1}\right)$ in five varieties of tomato (L. esculentum Mill.) at 30 days AS.

\begin{tabular}{|c|c|c|c|c|c|c|c|c|}
\hline \multirow{2}{*}{$\begin{array}{l}\text { Duration of } \\
\text { seed soaking } \\
\text { in Co }(\mu \mathrm{M})\end{array}$} & \multirow{2}{*}{ Treatments } & \multicolumn{6}{|c|}{ Varieties } & \multirow{2}{*}{ LSD at $5 \%$} \\
\hline & & K-25 & NTS-9 & NBR-Uday & Sarvodya & Malti & Mean & \\
\hline \multicolumn{2}{|c|}{ Unsoaked control } & 3.49 & 2.86 & 2.7 & 2.45 & 2.2 & 2.74 & \\
\hline & 0 & 3.52 & 2.99 & 2.73 & 2.5 & 2.23 & 2.79 & \\
\hline & 100 & 3.29 & 2.76 & 2.25 & 2.14 & 1.8 & 2.44 & $V=0.11$ \\
\hline \multirow[t]{5}{*}{$4 \mathrm{~h}$} & 200 & 2.99 & 2.38 & 2 & 2.05 & 1.32 & 2.14 & $\mathrm{C}=0.23$ \\
\hline & 300 & 2.74 & 1.97 & 1.69 & 1.5 & 1.19 & 1.81 & $\mathrm{VxC}=0.38$ \\
\hline & Mean & 3.13 & 2.52 & 2.16 & 2.04 & 1.63 & & \\
\hline & 0 & 3.56 & 3.01 & 2.74 & 2.53 & 2.25 & 2.83 & \\
\hline & 100 & 3.11 & 2.54 & 1.97 & 1.73 & 1.54 & 2.17 & $V=0.17$ \\
\hline \multirow[t]{5}{*}{$8 \mathrm{~h}$} & 200 & 2.7 & 2.29 & 1.74 & 1.5 & 1.3 & 1.9 & $\mathrm{C}=0.25$ \\
\hline & 300 & 2.36 & 1.77 & 1.5 & 1.25 & 1.17 & 1.6 & $\mathrm{VxC}=0.48$ \\
\hline & Mean & 2.93 & 2.4 & 1.98 & 1.75 & 1.56 & & \\
\hline & 0 & 3.56 & 3.02 & 2.77 & 2.54 & 2.26 & 2.83 & \\
\hline & 100 & 2.92 & 2.22 & 1.6 & 1.4 & 1.17 & 1.86 & $V=0.14$ \\
\hline \multirow[t]{3}{*}{$12 \mathrm{~h}$} & 200 & 2.5 & 1.8 & 1.39 & 1.15 & 1.11 & 1.59 & $C=0.20$ \\
\hline & 300 & 2.12 & 1.46 & 1.16 & 1.09 & 1.05 & 1.37 & $\mathrm{VxC}=0.39$ \\
\hline & Mean & 2.77 & 2.12 & 1.73 & 1.54 & 1.39 & & \\
\hline
\end{tabular}

$A S$, after sowing; $V$, varieties; $C$, cobalt concentration. 
Antioxidative enzymes and proline content: The data depicted in tables 10-13 clearly indicated that the activity of all the antioxidative enzymes and proline content exhibited a trend which was converse to that of all the other parameter explained earlier. The activity of antioxidative enzymes and proline content increased with the increasing concentration of metal as well as duration of soaking. The leaves of plants that were raised from the seeds received pre sowing seed soaking in 100,200 or $300 \mu \mathrm{M}$ of $\mathrm{Co}_{0}$ for $12 \mathrm{~h}$ possessed maximum enzyme activity for all the varieties. However, among variety, K-25 possessed maximum enzymatic activity as well as proline content in response to above said treatment. The values were closely followed by NTS-9 and NBR-Uday.

Table 10. Effect of pre sowing seed soaking treatment of cobalt $(100,200$ or $300 \mu \mathrm{M})$ for 4,8 or 12 h on the leaf CAT activity $\left[\mu\right.$ mol $\mathrm{H}_{2} \mathrm{O}_{2}$ decomposed g ${ }^{-1}(\mathrm{~F} . \mathrm{M}$.) $]$ in five varieties of tomato (L. esculentum Mill.) at 30 days AS.

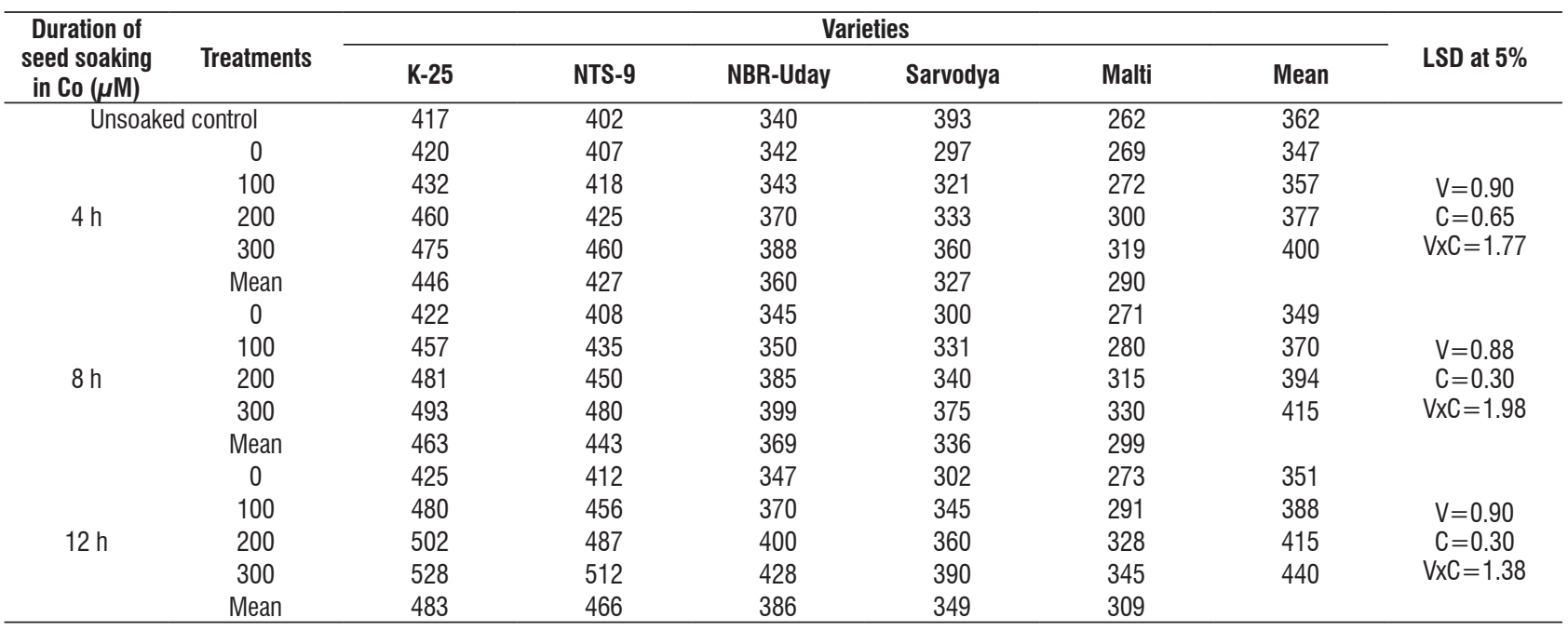

$A S$, after sowing; $V$, varieties; $C$, cobalt concentration.

Table 11. Effect of pre sowing seed soaking treatment of cobalt (100, 200 or $300 \mu \mathrm{M})$ for 4,8 or $12 \mathrm{~h}$ on the leaf POX activity (units $\mathrm{g}^{-1}$ (F.M.) in five varieties of tomato (L. esculentum Mill.) at 30 days AS.

\begin{tabular}{|c|c|c|c|c|c|c|c|c|}
\hline \multirow{2}{*}{$\begin{array}{l}\text { Duration of } \\
\text { seed soaking } \\
\text { in } \mathrm{Co}_{0}(\mu \mathrm{M})\end{array}$} & \multirow{2}{*}{ Treatments } & \multicolumn{6}{|c|}{ Varieties } & \multirow{2}{*}{ LSD at $5 \%$} \\
\hline & & K-25 & NTS-9 & NBR-Uday & Sarvodya & Malti & Mean & \\
\hline \multicolumn{2}{|c|}{ Unsoaked control } & 13.3 & 12.4 & 11.7 & 11.21 & 10.11 & 11.74 & \multirow{6}{*}{$\begin{array}{c}V=18.23 \\
C=9.10 \\
V x C=30.86\end{array}$} \\
\hline \multirow{5}{*}{$4 \mathrm{~h}$} & 0 & 13.3 & 12.45 & 11.72 & 11.24 & 10.1 & 11.76 & \\
\hline & 100 & 13.8 & 12.93 & 12.4 & 12 & 10.4 & 12.31 & \\
\hline & 200 & 14.3 & 13.8 & 12.63 & 12.09 & 10.63 & 12.69 & \\
\hline & 300 & 14.2 & 14.17 & 13.12 & 12.81 & 11.13 & \multirow[t]{2}{*}{13.17} & \\
\hline & Mean & 14 & 13.33 & 12.46 & 12.03 & 10.56 & & \\
\hline \multirow{5}{*}{$8 \mathrm{~h}$} & 0 & 13.4 & 12.5 & 11.77 & 11.29 & 11.13 & 11.81 & \multirow{5}{*}{$\begin{array}{c}V=18.00 \\
C=13.80 \\
V x C=36.44\end{array}$} \\
\hline & 100 & 13.9 & 13.1 & 12.38 & 11.6 & 10.52 & 12.3 & \\
\hline & 200 & 14.4 & 13.51 & 12.85 & 12.4 & 10.9 & 12.81 & \\
\hline & 300 & 14.9 & 14.16 & 13.4 & 12.63 & 11.29 & 13.28 & \\
\hline & Mean & 14.2 & 13.31 & 12.6 & 11.98 & 10.71 & & \\
\hline \multirow{5}{*}{$12 \mathrm{~h}$} & 0 & 13.4 & 12.51 & 11.79 & 11.32 & 11.16 & 13.03 & \multirow{5}{*}{$\begin{array}{c}V=15.39 \\
C=22.44 \\
V x C=43.39\end{array}$} \\
\hline & 100 & 14 & 13.41 & 12.66 & 11.79 & 10.63 & 12.49 & \\
\hline & 200 & 14.6 & 13.8 & 13 & 12.66 & 11 & 13.01 & \\
\hline & 300 & 15.1 & 14.26 & 13.71 & 12.74 & 11.35 & 13.43 & \\
\hline & Mean & 14.3 & 13.49 & 12.79 & 12.12 & 11.03 & & \\
\hline
\end{tabular}

AS, after sowing; $V$, varieties; $C$, cobalt concentration. 
Table 12. Effect of pre sowing seed soaking treatment of cobalt (100, 200 or $300 \mu \mathrm{M})$ for 4,8 or $12 \mathrm{~h}$ on the leaf SOD activity [units $\mathrm{g}^{-1}$ (F.M.)] in five varieties of tomato (L. esculentum Mill.) at 30 days AS.

\begin{tabular}{|c|c|c|c|c|c|c|c|c|}
\hline \multirow{2}{*}{$\begin{array}{l}\text { Duration of } \\
\text { seed soaking } \\
\text { in } \mathrm{Co}(\mu \mathrm{M})\end{array}$} & \multirow{2}{*}{ Treatments } & \multicolumn{6}{|c|}{ Varieties } & \multirow{2}{*}{ LSD at $5 \%$} \\
\hline & & K-25 & NTS-9 & NBR-Uday & Sarvodya & Malti & Mean & \\
\hline \multicolumn{2}{|c|}{ Unsoaked control } & 118 & 116 & 111 & 107 & 100 & 110 & \multirow{6}{*}{$\begin{aligned} V & =4.03 \\
C & =5.10 \\
V x C & =10.25\end{aligned}$} \\
\hline \multirow{5}{*}{$4 \mathrm{~h}$} & 0 & 125 & 121 & 113 & 110 & 105 & 114 & \\
\hline & 100 & 143 & 127 & 119 & 115 & 109 & 122 & \\
\hline & 200 & 151 & 139 & 126 & 122 & 113 & 130 & \\
\hline & 300 & 163 & 147 & 133 & 128 & 119 & \multirow[t]{2}{*}{138} & \\
\hline & Mean & 145 & 133 & 122 & 118 & 111 & & \\
\hline \multirow{5}{*}{$8 \mathrm{~h}$} & 0 & 127 & 122 & 115 & 112 & 104 & 116 & \multirow{5}{*}{$\begin{array}{c}V=4.60 \\
C=7.05 \\
V x C=12.59\end{array}$} \\
\hline & 100 & 147 & 135 & 125 & 120 & 113 & 128 & \\
\hline & 200 & 159 & 144 & 133 & 130 & 120 & 137 & \\
\hline & 300 & 168 & 160 & 154 & 145 & 128 & 151 & \\
\hline & Mean & 150 & 140 & 131 & 126 & 116 & & \\
\hline \multirow{5}{*}{$12 \mathrm{~h}$} & 0 & 128 & 124 & 116 & 115 & 106 & 117 & \multirow{5}{*}{$\begin{array}{c}V=7.40 \\
C=5.32 \\
V x C=13.80\end{array}$} \\
\hline & 100 & 164 & 146 & 139 & 128 & 120 & 139 & \\
\hline & 200 & 173 & 160 & 147 & 135 & 124 & 147 & \\
\hline & 300 & 179 & 173 & 160 & 140 & 119 & 154 & \\
\hline & Mean & 161 & 150 & 140 & 129 & 117 & & \\
\hline
\end{tabular}

$A S$, after sowing; $V$, varieties; $C$, cobalt concentration.

Table 13. Effect of pre sowing seed soaking treatment of cobalt (100, 200 or $300 \mu \mathrm{M})$ for 4,8 or $12 \mathrm{~h}$ on the leaf proline content $\left[\mu \mathrm{mol} \mathrm{g}^{-1}\right.$ (F.M.)] in five varieties of tomato (L. esculentum Mill.) at 30 days AS.

\begin{tabular}{|c|c|c|c|c|c|c|c|c|}
\hline \multirow{2}{*}{$\begin{array}{l}\text { Duration of } \\
\text { seed soaking } \\
\text { in } \mathrm{Co}(\mu \mathrm{M})\end{array}$} & \multirow{2}{*}{ Treatments } & \multicolumn{6}{|c|}{ Varieties } & \multirow{2}{*}{ LSD at $5 \%$} \\
\hline & & K-25 & NTS-9 & NBR-Uday & Sarvodya & Malti & Mean & \\
\hline \multicolumn{2}{|c|}{ Unsoaked control } & 9.59 & 7.7 & 6.32 & 5.7 & 4.82 & 6.82 & \multirow{6}{*}{$\begin{array}{c}V=0.40 \\
C=0.29 \\
V x C=0.78\end{array}$} \\
\hline \multirow{5}{*}{$4 \mathrm{~h}$} & 0 & 9.61 & 7.77 & 6.38 & 5.77 & 4.86 & 6.87 & \\
\hline & 100 & 11.5 & 9.89 & 7.9 & 6.1 & 5.02 & 8.08 & \\
\hline & 200 & 16.1 & 11.22 & 8.22 & 6.59 & 5.68 & 9.56 & \\
\hline & 300 & 19 & 14.06 & 8.93 & 6.88 & 5.99 & \multirow[t]{2}{*}{10.97} & \\
\hline & Mean & 14.1 & 10.73 & 7.85 & 6.33 & 5.38 & & \\
\hline \multirow{5}{*}{$8 \mathrm{~h}$} & 0 & 9.89 & 7.9 & 6.66 & 5.89 & 4.99 & 7.06 & \multirow{5}{*}{$\begin{array}{c}V=0.60 \\
C=0.37 \\
V x C=1.11\end{array}$} \\
\hline & 100 & 12 & 10.21 & 8.11 & 6.2 & 5.1 & 8.32 & \\
\hline & 200 & 16.5 & 11.99 & 8.8 & 7 & 6 & 10.05 & \\
\hline & 300 & 19.9 & 14.9 & 10.04 & 7.15 & 6.41 & 11.68 & \\
\hline & Mean & 4.58 & 11.25 & 8.4 & 6.56 & 5.62 & & \\
\hline \multirow{5}{*}{$12 \mathrm{~h}$} & 0 & 10 & 8.1 & 6.79 & 5.95 & 5.08 & 7.18 & \multirow{5}{*}{$\begin{array}{c}V=0.65 \\
C=0.34 \\
V x C=1.13\end{array}$} \\
\hline & 100 & 13 & 10.54 & 8.46 & 6.32 & 5.3 & 8.72 & \\
\hline & 200 & 18.1 & 13.81 & 9.17 & 7.16 & 6.21 & 10.89 & \\
\hline & 300 & 21.6 & 17.11 & 10.3 & 7.49 & 6.53 & 11.21 & \\
\hline & Mean & 15.7 & 12.39 & 8.68 & 6.7 & 5.78 & & \\
\hline
\end{tabular}

$A S$, after sowing; $V$, varieties; $C$, cobalt concentration. 


\section{DISCUSSION}

Heavy metals are well known, to generate a large quantity of reactive oxygen species (ROS) in plants that may oxidize protein, lipids and nucleic acid resulting to the abnormalities at the level of the cell (Sanita di Toppi et al., 1999). To maintain metabolic functions under stress conditions, the balance between generation and degeneration of ROS is required; for this purpose plants have well equipped antioxidant system that include antioxidant enzymes (superoxide dismutase, catalase, peroxidase and glutathione reductase) and nonenzymatic low molecular weight antioxidants (glutathione, proline, carotenoids, tocopherols etc.) (Schutzendubell and Polle, 2002). Therefore, an observed increased in the antioxidant enzymes (Tables 10-12) i.e. CAT, POS, SOD and that of proline content (Table 13) in different cultivars of tomato in the present study is a biochemical adaptation of these cultivars to protect themselves against the oxidative stress that burst out in response to Co stress. The literature of the recent years strongly favors this fact further as an increased in the antioxidant system was observed when the plants were exposed to Co (Chatterjee et al., 2006), nickel (Alam et al., 2007), cadmium (Hasan et al., 2008; Hayat et al., 2009), aluminum (Ali et al., 2008) and copper (Fariduddin et al., 2009).

The present investigation reveals that $\mathrm{Co}$ stress caused a significant reduction in the NR activity of tomato cultivars. The possible reason that may simplify the cause behind this reduction may be the restriction of $\mathrm{NO}_{2}$ uptake (Hernandez et al., 1996), the inducer and substrate of NR (Campbell, 1999), as it is a well established fact that Co interfere with the uptake and transport of nutrients (Liu et al., 2000). In addition to this, our results are in consistent with those of Ali et al. (2008) and Hayat et al. (2007), who emphasized that heavy metals sharply decreased $\mathrm{NO}_{3}$ uptake.

Carbonic anhydrase (CA) is the enzyme that catalyzes interconversion of $\mathrm{CO}_{2}$ and $\mathrm{HCO}_{3}{ }^{-}$and its activity is largely determined by photon flux density, concentration of $\mathrm{CO}_{2}$, availability of Zn (Tiwari et al., 2005) and the genetic expression (Kim et al., 1994). The stress generated by Co decreased the internal $\mathrm{CO}_{2}$ concentration (Table 7) and interfere with the availability of Zn (Liu et al., 2000). The cumulative effect of these factors resulted in a decrease in the activity of CA (Table 9).
It is also evident from the present study that SPAD chlorophyll content (Table 5) and the photosynthetic attributes (Tables 6-7) significantly decreased in the tomato cultivars on being fed with $\mathrm{Co}$ before sowing. Heavy metals is known to enhance the level of the enzyme chlorophyllase that bring about the degradation of the chlorophyll (Reddy and Vora, 1986) and in particular Co inhibit the activity of the enzymes that involved in the synthesis of chlorophyll synthesis, such as 5-aminolevulinic acid and protoporphyrin (Shalygo et al., 1999). Similar, observations have also been reported earlier in tomato (Gopal et al., 2003) and French beans (Chatterjee et al., 2006) under $\mathrm{Co}$ stress. The stress generated by $\mathrm{Co}$ also caused a marked reduction in net photosynthetic rate in all the tomato cultivar that may be a direct outcome of reduced stomatal conductance and internal $\mathrm{CO}_{2}$ concentration (Tables 6-7) in addition to decreased photosynthetic pigment and activity of carbonic anhydrase. The reason behind such belief is the study of Mysliva-Kurdziel et al. (2004) who suggested that heavy metal affect the photosynthetic machinery at multiple levels such as pigment biosynthesis/ degradation, stomatal functioning enzyme inhibition, alteration in membrane structure/ function and photosystem. Decreased in the leaf water potential (Table 14) in the present study in response to Co toxicity may be justify on the ground of physiological drought that was generated by heavy metals (Barcelo and Poschenrieder, 1990).

All these physiological disorders are further expressed in the form of reduced growth (Tables 1-3). The decrease in the growth attributes (fresh and dry mass of shoot and leaf area) is a direct outcome of inhibition of cell division or cell elongation, or a combination of both under Co stress (Jayakumar et al., 2007). Earlier reports also favor this finding that Co toxicity generates marked reduction in growth attributes in radish (Jayakumar et al., 2007).

\section{CONCLUSION}

It is concluded from the present study that despite substantial varietal difference among tomato cultivars against $\mathrm{Co}$ toxicity, $\mathrm{Co}$ induced marked reduction in growth, photosynthesis, and the activity of NR and CA in all the varieties. In addition to this, antioxidative enzymatic activity (POX, CAT, SOD) and proline content emerged out as a good markers for estimating the degree of tolerance among 
tomato cultivars, against the toxicity generated by $\mathrm{Co}$ in the present study.

Acknowledgement: The award of Senior Research Fellowship by CSIR, New Delhi, India to Ms. Syed Aiman Hasan is gratefully acknowledged.

\section{REFERENCES}

Alam MM, Hayat S, Ali B, Ahmad A (2007) Effect of 28-homobrassinolide on nickel induced changes in Brassica juncea. Photosynthetica 45:139142.

Ali B, Hasan SA, Hayat S, Hayat Q, Yadav S, Fariduddin Q, Ahmad A (2008) A role of brassinosteroids in the amelioration of aluminium stress through antioxidant system in mung bean (Vigna radiata L.Wilczek). Environ. Exp. Bot. 62:153-159.

Barcelo J, Poschenrieder C (1990) Plant water relations as affected by heavy metals: a review. J Plant Nutr. 13:1-37.

Bates LS, Walden RT, Tearse ID (1973) Rapid determination of free proline for water stress studies. Plant Soil 39: 205-207.

Beauchamp LO, Fridovich I (1971) Superoxide dismutase improved assays and assay applicable to acrylamide gels. Annals Biochem. 44:276-287

Campbell HW (1999) Nitrate reductase structure, function and regulation bridging the gap between biochemistry and physiology. Ann. Rev. Plant Physiol. Plant Mol. Biol. 50:277-303.

Chance B, Maehly AC (1955) Assay of catalase and peroxidase. Methods Enzymol. 2:764-775.

Chatterjee C, Gopal R, Dube BK (2006) Physiological and biochemical responses of French beans to excess cobalt. J. Plant Nutr. 29:127-136.

Dubey RS, Dwivedi RS (1987) Effect of heavy metal on seed germination and seedling growth of soybean. Nat. Acad. Sci. Letter 10:121-124.

Dwivedi RS, Randhawa NS (1974) Evolution of a rapid test of the hidden hunger of zinc in plants. Plant Soil 40:445-451.

Fariduddin Q, Khannam S, Hasan SA, Ali B, Hayat S, Ahmad A (2009) Effect of 28-homobrassinolide on the drought stress-induced changes in photosynthesis and antioxidant system of Brassica juncea. L. Acta Physiol. Plant 31:889-897.

Gopal R, Dube BK, Sinha P, Chatterjee C (2003) Cobalt toxicity effect on growth and metabolism of tomato. Commun. Soil Sci. Plant Anal. 34:619-628.

Hasan SA, Hayat S, Ali B, Ahmad A (2008) 28-homobrassinolide protect chickpea (Cicer arietinum) from cadmium toxicity by stimulating antioxidant. Environ. Poll. 151:60-66.

Hayat S, Ali B, Hasan SA, Ahmad A (2007) Brassinosteroid enhanced the level of antioxidants under cadmium stress in Brassica juncea. Environ. Exp. Bot. 60:33-41.
Hayat S, Hasan SA, Hayat Q, Ahmad A (2010) Brassinosteroids protects Lycopersicon esculentum from cadmium toxicity applied as shotgun approach. Protoplasma 239:3-14.

Hernandez LE, Carpena-Ruiz R, Garate A (1996) Alterations in the mineral nutrition of pea seedlings in exposed to cadmium. J. Plant Nutr. 19:15811598.

Jaworski EG (1971) Nitrate reductase assay in intact plant tissues. Biochem. Biophys. Res. Commun. 43:1274-1279.

Jayakumar K, Jaleel CA, Vijayarengan $P$ (2007) Changes in growth, biochemical constituent and antioxidant potentials in radish (Raphanus sativus L.) under cobalt stress. Turkish J. Biol. 31:127-136.

Jayakumar K, Vijayarengan P (2006) Alterations in the carbohydrate metabolism of Vigna mungo (L.) Hepper as affected by cobalt stress. Ind. J. Environ. Ecoplan. 12:693-696.

Kabata-Pendias A, Pendias H (1991) Trace elements in soils and plants. CRC, Boca Raton, FL. pp. 276-285.

Kim HJ, Bracey MH, Barlett SG (1994) Nucleotide sequence of a gene encoding carbonic anhydrase in Arabidopsis thaliana. Plant Physiol. 105:449-450.

Liu J, Reid RJ, Smith FA (2000) The mechanism of cobalt toxicity in mung beans. Physiol. Plant 110:104-110.

Mysliva-Kurdziel B, Prasad MNV, Strzalka K (2004) Photosynthesis in metal stressed plants. In: MNV Prasad (Eds) Heavy metal stress in plants: from biomolecule to ecosystems, Springer-Verlag, Berlin pp. 146-181.

Reddy MP, Vora AB (1986) Changes in pigment composition, hill reaction activity and saccharide metabolism in bajra (Pennisetum typhoides $\mathrm{S} \& \mathrm{H})$ leaves under $\mathrm{NaCl}$ salinity. Photosynthetica 20:50-55.

Sanita di TL, Gabbrielli R (1999) Response of cadmium in higher plant. Environ. Exp. Bot. 41:105-130.

Schutzendubell A, Polle A (2002) Plant responses to abiotic stresses: heavy metal-induced oxidative stress and protection by mycorrhization. J. Exp. Bot. 53:1351-1365.

Shalygo NV, Kolesnikova NV, Voronetskaya VV, Averina NG (1999) Effect of $\mathrm{Mn}^{2+}, \mathrm{Fe}^{2+}, \mathrm{Co}^{2+}$, and $\mathrm{Ni}^{2+}$ on cholorophyll accumulation and early stages of cholorophyll formation in greening barley seedlings. Russ. J. Plant Physiol. 46:496-501.

Tiwari A, Kumar P, Singh S, Ansari SA (2005) Carbonic anhydrase in relation to higher plants. Photosynthetica 43:1-9.

Van Assche F, Clijsters H (1990) Effects of metals on enzyme activity in plant. Plant Cell Environ. 13:195-206.

Vanselow AP (1966) Cobalt. In Diadnostic criteria of plants and soils, ed. HD Chapman, 142-156. Riverside, CA: University of California Division of Agricultural Science.

Young RS (1979) Cobalt in biology and biochemistry. Academic Press. London pp.52-97. 